\title{
Totally real origami and impossible paper folding
}

\author{
David Auckly and John Cleveland \\ Department of Mathematics \\ The University of Texas at Austin \\ Austin, TX 78712
}

\begin{abstract}
Origami is the ancient Japanese art of paper folding. It is possible to fold many intriguing geometrical shapes with paper $[\mathrm{M}]$. In this article, the question we will answer is which shapes are possible to construct and which shapes are impossible to construct using origami. One of the most interesting things we discovered is that it is impossible to construct a cube with twice the volume of a given cube using origami, just as it is impossible to do using a compass and straight edge. As an unexpected surprise, our algebraic characterization of origami is related to David Hilbert's $17^{\text {th }}$ problem. Hilbert's problem is to show that any rational function which is always non-negative is a sum of squares of rational functions [B]. This problem was solved by Artin in 1926 [Ar]. We would like to thank John Tate for noticing the relationship between our present work and Hilbert's $17^{\text {th }}$ problem. This research is the result of a project in the Junior Fellows Program at The University of Texas. The Junior Fellows Program is a program in which a junior undergraduate strives to do original research under the guidance of a faculty mentor.

The referee mentioned two references which the reader may find interesting. "Geometric Exercises in Paper Folding" addresses practical problems of paper folding $[\mathrm{R}]$. Among many other things, Sundara Row gives constructions for the 5-gon, the 17-gon, and duplicating a cube. His constructions, however, use more general folding techniques than the ones we consider here. Felix Klein cites Row's work in his lectures on selected questions in elementary geometry $[\mathrm{K}]$.

In order to understand the rules of origami construction, we will first consider a sheet of everyday notebook paper. Our work with notebook paper will serve as an intuitive model for our definition of origami constructions in the Euclidean plane. There are four
\end{abstract}


natural methods of folding a piece of paper. The methods will serve as the basis of the definition of an origami pair.

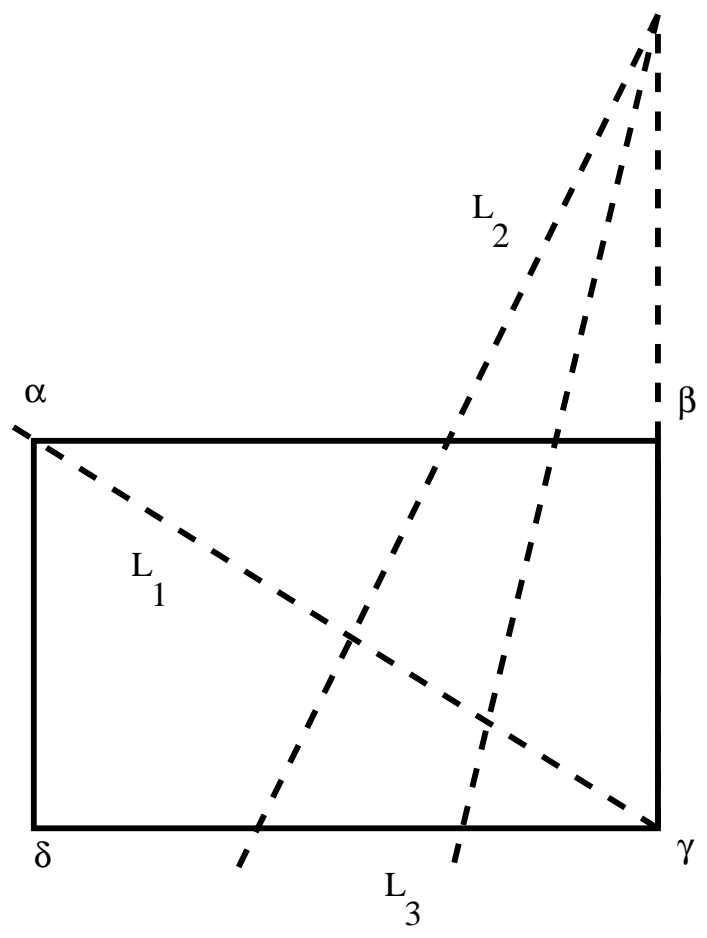

Figure 1

We construct the line $L_{1}$, by folding a crease between two different corners of the paper. Another line may be constructed by matching two corners. For example, if corners $\alpha$ and $\gamma$ are matched, the crease formed, $L_{2}$, will be the perpendicular bisector of the segment $\overline{\alpha \gamma}$. another natural construction is matching one line to another line. For instance, $\overline{\beta \gamma}$, the paper's edge, and $L_{2}$ are lines. If we lay $\overline{\beta \gamma}$ upon $L_{2}$ and form the crease, then we obtain $L_{3}$ which is the angle bisector of the two lines. If we start with two parallel lines in this third construction, then we will just get a parallel line half way in between.

The fourth and final construction which seems natural is consecutive folding. This is similar to rolling up the sheet of paper only one does not roll it up, he folds it up. More explicitly, start with a piece of paper with two creases on it as in figure 2. Fold along line $L_{1}$ and do not unfold the piece of paper. Notice that line $L_{2}$ lies over the sheet of paper. With the paper still folded, fold the sheet of paper along the crease $L_{2}$ to obtain a new crease on the sheet underneath $L_{2}$. If we name this new crease $L_{3}$ and unfold the sheet of paper, then it is easy to see that line $L_{3}$ is the mirror image or reflection of line $L_{2}$ about line $L_{1}$. 


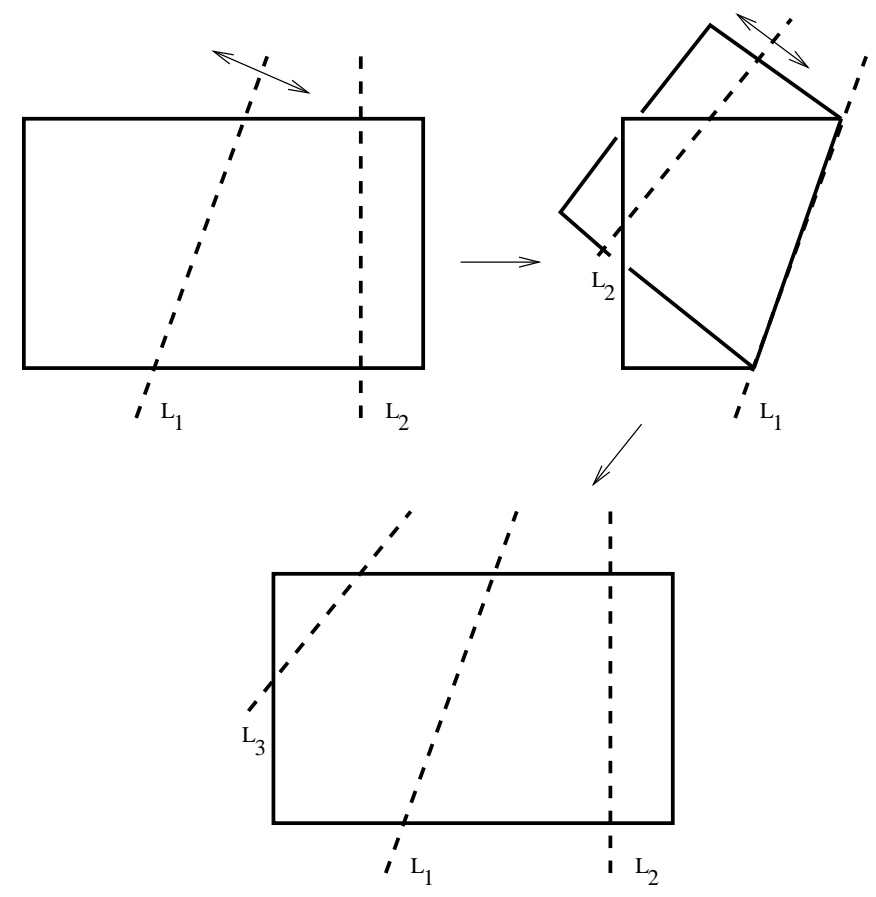

Figure 2

We now formalize these methods to define an origami pair on the plane. The creases on our sheet of paper are merely lines in the plane, and the corners of the paper are represented by points where lines (creases) meet. This previous discussion is the motivation for the following definition.

Definition. $(\mathcal{P}, \mathcal{L})$ is an origami pair if $\mathcal{P}$ is a set of points in $\mathbb{R}^{2}$ and $\mathcal{L}$ is a collection of lines in $\mathbb{R}^{2}$ satisfying:

i) The point of intersection of any two non-parallel lines in $\mathcal{L}$ is a point in $\mathcal{P}$.

ii) Given any two distinct points in $\mathcal{P}$, there is a line in $\mathcal{L}$ going through them.

iii) Given any two distinct points in $\mathcal{P}$, the perpendicular bisector of the line segment with given end points is a line in $\mathcal{L}$.

iv) If $L_{1}$ and $L_{2}$ are lines in $\mathcal{L}$, then the line which is equidistant from $L_{1}$ and $L_{2}$ is in $\mathcal{L}$.

v) If $L_{1}$ and $L_{2}$ are lines in $\mathcal{L}$, then there exists a line $L_{3}$ in $\mathcal{L}$ such that $L_{3}$ is the mirror reflection of $L_{2}$ about $L_{1}$.

For any subset of the plane containing at least two points, there is at most one collection of lines which will pair with it to become an origami pair. 
Definition. A subset of $\mathbb{R}^{2}, \mathcal{P}$, is closed under origami constructions if there exists a collection of lines, $\mathcal{L}$, such that $(\mathcal{P}, \mathcal{L})$ is an origami pair.

The question which we answer in this paper is which points may be constructed from just two points, using only the origami constructions described above. We will call that collection of points the set of origami constructible points.

Definition. $\quad \mathcal{P}_{0}=\cap\{\mathcal{P} \mid(0,0),(0,1) \in \mathcal{P}$ and $\mathcal{P}$ is closed under origami constructions $\}$ is the set of origami constructible points.

Before we explain the structure of $\mathcal{P}_{0}$, we give an example of an origami construction analogous to many compass and straight edge constructions, namely, the construction of parallel lines.

Lemma. It is possible to construct a line parallel to a given line through any given point using origami.

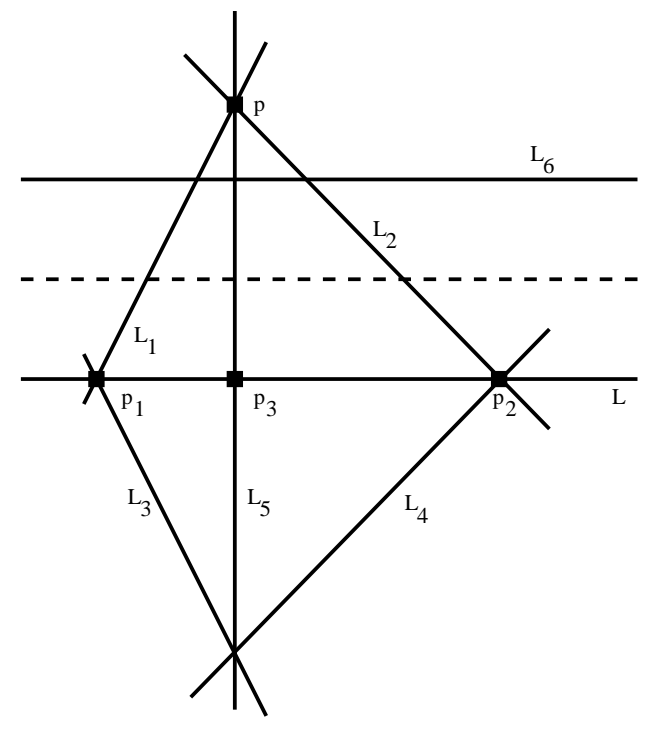

Figure 3

Proof. Refer to Figure 3. Given a line $L$ and a point $p$, pick two points $p_{1}$ and $p_{2}$ on $L$. By property ii) in the definition of an origami pair, we may construct lines $L_{1}$ and $L_{2}$ running through $p_{1}, p$ and $p_{2}, p$, respectively. By property v) we may reflect $L_{1}$ and $L_{2}$ through $L$ to obtain $L_{3}$ and $L_{4}$. Now the intersection of $L_{3}$ and $L_{4}$ is a constructible point, so there is a line, $L_{5}$ through this point and the given point, $p$, by properties i) and ii). Call the point where $L_{5}$ and $L$ intersect $p_{3}$. To finish the construction, use property iii) 
to construct a perpendicular bisector to $\overline{p, p_{3}}$, and reflect $L$ through this bisector with property v) to obtain the desired line, $L_{6}$. It is a straightforward exercise to show that $L_{6}$ has the desired properties.

The reader may wish to try some constructions on his own. Two especially interesting exercises to attempt are the construction of a right triangle with given legs and the construction of a right triangle with a given hypotenuse and leg. More explicitly, given four distinct points $\alpha, \beta, \gamma$ and $\delta$, the reader may try to construct a point $\varepsilon$ such that $\alpha, \beta, \varepsilon$ are the vertices of a right triangle with legs $\overline{\alpha \beta}$ and $\overline{\beta \varepsilon}$ such that the length of $\overline{\beta \varepsilon}$ equals the length of $\overline{\gamma \delta}$.

Now that we have a better feel for origami constructions, we will start developing tools to show that some figures are not constructible. The first thing we need is the notion of an origami number.

Definition. $\mathbb{F}_{0}=\left\{\alpha \in \mathbb{R} \mid \exists v_{1}, v_{2} \in \mathcal{P}\right.$ such that $\left.|\alpha|=\operatorname{dist}\left(v_{1}, v_{2}\right)\right\}$ is the set of origami numbers.

It is easy to see that $(x, y) \in \mathcal{P}_{0}$ if and only if $x$ and $y$ are both in $\mathbb{F}_{0}$. It is also easy to see that the numbers $\frac{1}{2}, \frac{1}{4}, \frac{1}{8}, \ldots$ are origami numbers. To see that $\frac{1}{5}$ is an origami number consider figure 4 .

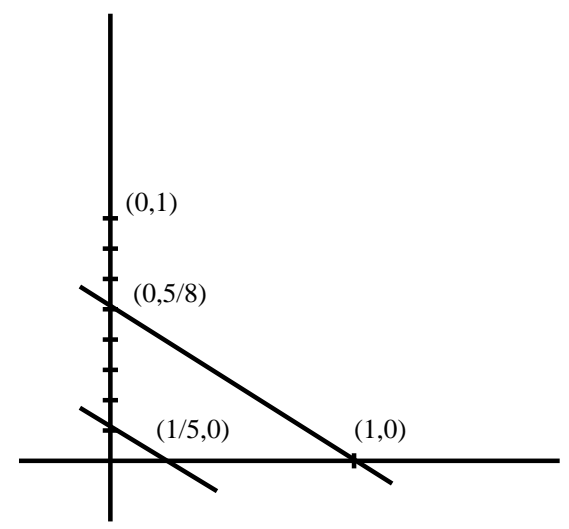

Figure 4

In figure 4 a line through $\left(0, \frac{5}{8}\right)$ and $(1,0)$ is constructed, then a parallel line through $\left(0, \frac{1}{8}\right)$ is constructed. This parallel line intersects the $x$-axis at $\left(\frac{1}{5}, 0\right)$, therefore $\frac{1}{5}$ is an origami number. Another class of origami numbers can be generalized by a simple geometric construction. Starting with any segment, it is possible to construct a right triangle as in figure 5. 


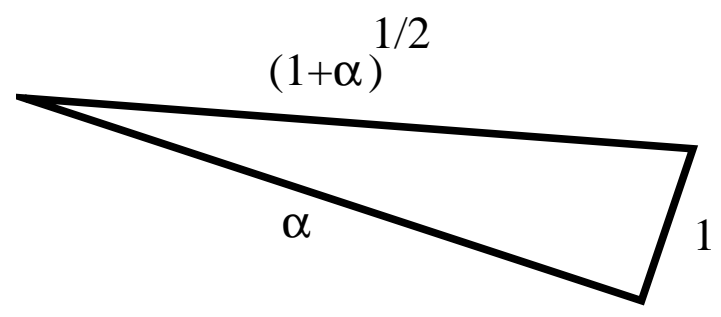

Figure 5

It follows that $\sqrt{1+\alpha^{2}}$ is an origami number whenever $\alpha$ is an origami number. Using this construction, we see that

$$
\sqrt{2}=\sqrt{1+1^{2}} \text { and } \quad \sqrt{3}=\sqrt{1+(\sqrt{2})^{2}}
$$

are origami numbers. In fact, the sum, difference, product, and quotient of origami numbers are origami numbers.

Theorem. The collection of origami numbers, $\mathbb{F}_{0}$ is a field closed under the operation $\alpha \mapsto \sqrt{1+\alpha^{2}}$.

Proof. If $\alpha, \beta \in \mathbb{F}_{0}$, it follows from the definition that $-\alpha \in \mathbb{F}_{0}$ and it is easy to show that $\alpha+\beta \in \mathbb{F}_{0}$. Straightforward constructions with similar triangles are enough to show that $\alpha \cdot \beta, \alpha^{-1} \in \mathbb{F}_{0}$. See figure 6 .
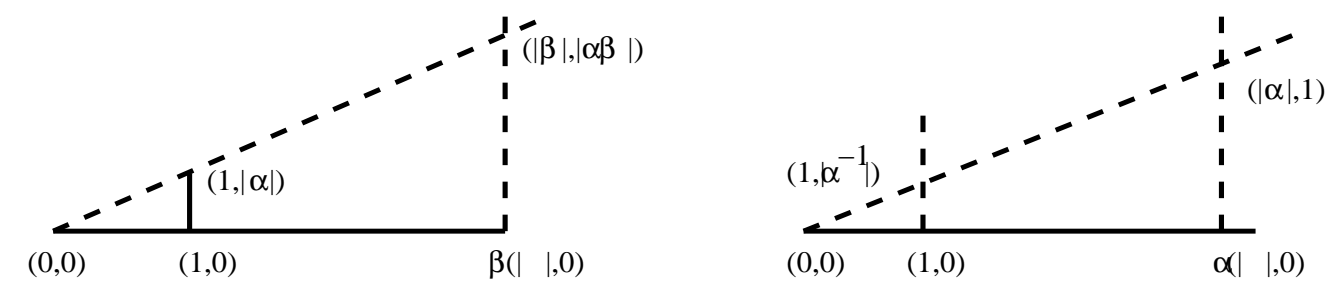

Figure 6

In the discussion preceding this theorem we showed that $\sqrt{1+\alpha^{2}}$ is an origami number whenever $\alpha$ is. The proof is therefore complete.

Now that we have some algebraic operations which will produce origami numbers, it is natural to ask if there are any more operations which will produce origami numbers. Once we have a list of all ways to create origami numbers and a method to test if a given number can be achieved, then we will know which geometric shapes are constructible, and which shapes are not constructible. This is because any figure is constructible if and only if the coordinates of all of the vertices are origami numbers. 
Definition. $\quad \mathbb{F}_{\sqrt{1+x^{2}}}$ is the smallest subfield of $\mathbb{C}$ closed under the operation $x \mapsto \sqrt{1+x^{2}}$.

The preceding Theorem may be rephrased as $\mathbb{F}_{\sqrt{1+x^{2}}} \subset \mathbb{F}_{0}$. It is in fact true that $\mathbb{F}_{0}=\mathbb{F}_{\sqrt{1+x^{2}}}$. Thus, the previously listed operations which produce origami numbers are the only independent operations which produce origami numbers.

Theorem. $\mathbb{F}_{0}=\mathbb{F}_{\sqrt{1+x^{2}}}$.

Proof. Since we already know that $\mathbb{F}_{\sqrt{1+x^{2}}} \subset \mathbb{F}_{0}$, we only need to show that $\mathbb{F}_{0} \subset \mathbb{F}_{\sqrt{1+x^{2}}}$. That is, we need to show that any origami number may be expressed using the usual field operations and the operation $x \mapsto \sqrt{1+x^{2}}$. It is enough to consider the coordinates of origami constructible points, because a number is an origami number if and only if it is a coordinate of a constructible point. There are only four distinct ways of constructing new origami points from old ones using the axioms for origami construction. These are illustrated in figures 7 and 8 .
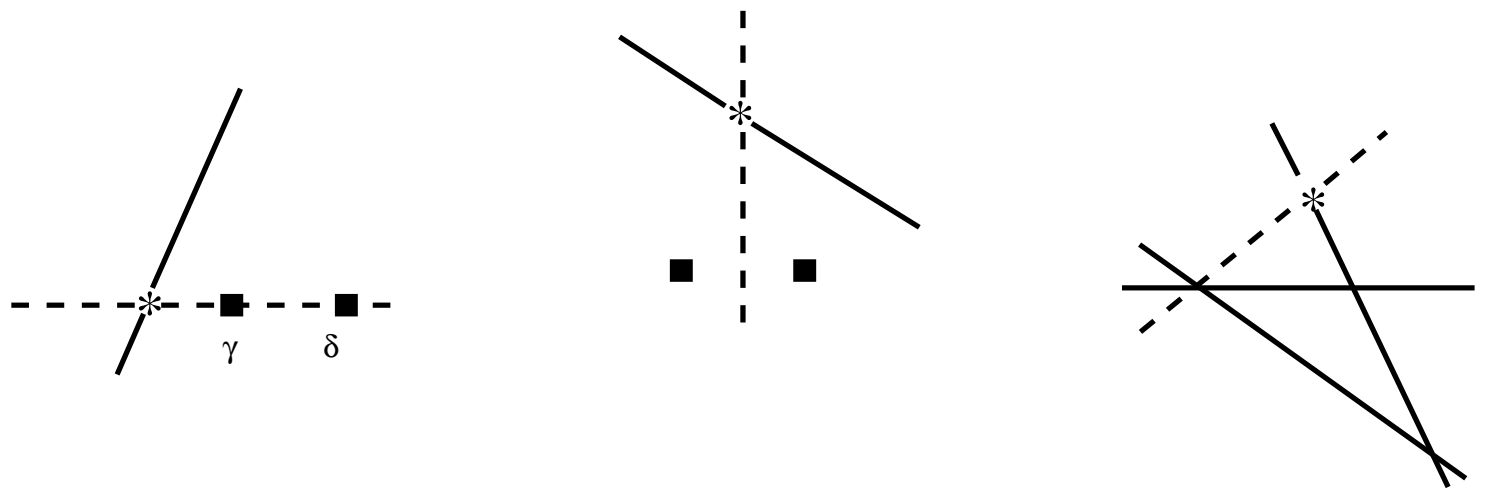

Figure 7

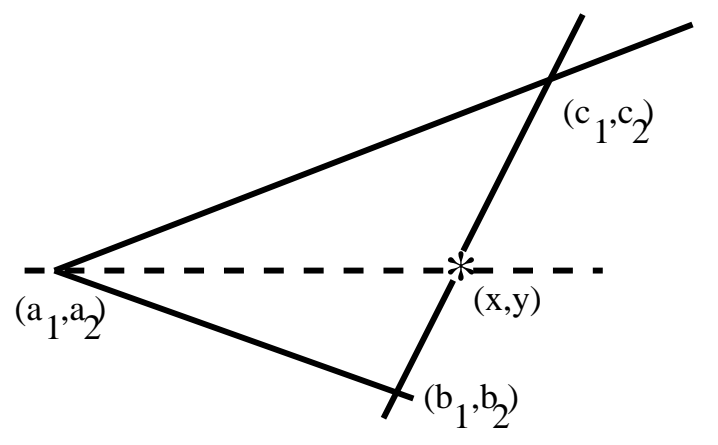

Figure 8

The only way a new point will be constructed is by a new crease intersecting an old one. The four ways of making a crease are: folding a line between two existing points as in the 
line $\overline{\gamma \delta}$ in figure 7 , folding the perpendicular bisector to two points as in the second part of figure 7, reflecting a line as in the third part of figure 7, or forming the angle bisector as in figure 8. We will explain the case illustrated in figure 8 and leave the remaining three cases to the reader. When showing that the point $(x, y)$ only depends on the prescribed operations, we may assume that $\left(a_{1}, a_{2}\right)=(0,0)$ by translation, because the point $(x, y)$ is found by adding $\left(a_{1}, a_{2}\right)$ to the translated point. We may further assume that $\left(b_{1}, b_{2}\right)$ is on the unit circle, by scaling because multiplying by $\sqrt{b_{1}^{2}+b_{2}^{2}}=\left|b_{1}\right| \sqrt{1+\left(b_{2} / b_{1}\right)^{2}}$ will reverse the scaling. Even further $\left(b_{1}, b_{2}\right)$ may be assumed to be $(1,0)$ because the rotation $(x, y) \mapsto\left(b_{1} x-b_{2} y, b_{2} x+b_{1} y\right)$ sends the point $(1,0)$ back to $\left(b_{1}, b_{2}\right)$. Let $\theta=\angle c a b$, with the above assumptions, $\cot \theta=c_{1} / c_{2}$ and $\csc \theta=\sqrt{c_{1}^{2}+c_{2}^{2}} / c_{2}=\sqrt{1+\left(c_{1} / c_{2}\right)^{2}}$. Now the slope of the new crease is $m=\tan (\theta / 2)=\csc \theta-\cot \theta$, which only depends on the prescribed operations. The new point $(x, y)$ is the intersection of the two lines $y=m x$ and $y=\left[c_{2} /\left(c_{1}-1\right)\right](x-1)$, so

$$
x=\frac{c_{2}}{c_{2}-m\left(c_{1}-1\right)} \quad \text { and } \quad y=\frac{m c_{2}}{c_{2}-m\left(c_{1}-1\right)}
$$

which only depends on the prescribed operations as was to be shown.

The preceding theorem gives an algebraic description of the field of origami numbers, and in principle answers which shapes are constructible and which are not constructible using origami. In practice it is still difficult to decide whether or not a given number is an origami number. For example, $\sqrt{4+2 \sqrt{2}}$ is an origami number because $\sqrt{4+2 \sqrt{2}}=$ $\sqrt{1+(1+\sqrt{2})^{2}}$, but what about $\sqrt{1+\sqrt{2}}$ ? In order to answer this question we need a better characterization of origami numbers. Before we proceed we will review some elementary facts from abstract algebra $[\mathrm{AH}],[\mathrm{L}]$.

Definition. A number, $\alpha$, is an algebraic number if it is a root of a polynomial with rational coefficients.

Any algebraic number, $\alpha$, is a root of a unique monic irreducible polynomial in $\mathbb{Q}[x]$, denoted by $p_{\alpha}(x)$. This polynomial, moreover, divides any polynomial in $\mathbb{Q}[x]$ having $\alpha$ as a root.

Definition. The conjugates of $\alpha$ are the roots of the polynomial $p_{\alpha}(x)$. An algebraic number is totally real if all of its conjugates are real. We denote the set of totally real numbers by $\mathbb{F}_{T R}$. 
Of the numbers which we are using to motivate this section, $\sqrt{4+2 \sqrt{2}}$ is totally real, because all of its conjugates $( \pm \sqrt{4 \pm 2 \sqrt{2}})$ are real, but $\sqrt{1+\sqrt{2}}$ is not totally real because two of its conjugates are imaginary $( \pm \sqrt{1-\sqrt{2}})$.

The last topic which we review is symmetric polynomials. The symmetric group on $n$ letters acts on polynomials in $n$ variables by $\sigma f\left(x_{1}, x_{2}, \ldots, x_{n}\right)=f\left(x_{\sigma(1)}, x_{\sigma(2)}, \ldots, x_{\sigma(n)}\right)$ where $f \in R\left[x_{1}, \ldots, x_{n}\right]$ and $R$ is an arbitrary ring.

Definition. The fixed points of the above action are called symmetric polynomials over $R$.

For example, $x_{1}^{2}+x_{2}^{2}$ is a symmetric polynomial in two variables because it remains unchanged when the variables are interchanged. However, $x_{1}^{2}-x_{2}^{2}$ is not a symmetric polynomial because it becomes $x_{2}^{2}-x_{1}^{2} \neq x_{1}^{2}-x_{2}^{2}$ when $x_{1}$ and $x_{2}$ are interchanged. One important class of symmetric polynomials is the class of elementary symmetric polynomials.

Definition. If $\prod_{k=1}^{n}\left(t+x_{k}\right)$ is expanded, we obtain

$$
\prod_{k=1}^{n}\left(t+x_{k}\right)=\sum_{\ell=0}^{n} \sigma_{\ell}\left(x_{1}, \ldots, x_{n}\right) t^{n-\ell}
$$

The $\sigma_{\ell}\left(x_{1}, \ldots, x_{n}\right)$ are the elementary symmetric polynomials.

It is easily verify that

$$
\begin{aligned}
\sigma_{1} & =x_{1}+x_{2}+\cdots+x_{n} \\
\sigma_{\ell} & =\text { the sum of all products of } \ell \text { distinct } x_{k} \text { 's } \\
\sigma_{n} & =x_{1} \cdot x_{2} \cdots x_{n} .
\end{aligned}
$$

Fact. [L, page 191]. The algebra of symmetric polynomials over $R$ is generated by the elementary symmetric polynomials. That is, any symmetric polynomial is a linear combination of products of the elementary symmetric polynomials.

We will now begin the final characterization of the origami numbers. It happens that all origami numbers are totally real. To prove this, it is necessary to show that the sum, difference, product and quotient of totally real numbers is totally real, and that $\sqrt{1+\alpha^{2}}$ is totally real whenever $\alpha$ is totally real. This is proven by using symmetric polynomials and the following lemma. 
Lemma.

$$
\prod_{i=1}^{n} \prod_{j=1}^{m}\left(t-x_{i} y_{j}\right)=\operatorname{det}(t I-A B)
$$

where $A$ and $B$ are matrices with entries expressed in terms of the elementary symmetric polynomials of $x_{i}$ or $y_{j}$ respectively.

This lemma is interesting because it is easier to prove a more general statement which implies the lemma than it is to verify the lemma. We will prove the lemma when the $x_{i}$ and $y_{j}$ are independent variables, a more general statement than when the $x_{i}$ and $y_{j}$ represent numbers, but, nevertheless, an easier statement to prove.

Proof. Let

$$
P_{A}(t)=\prod_{k=1}^{n}\left(t-x_{k}\right)=\sum_{\ell=0}^{n}(-1)^{\ell} \sigma_{\ell}(\mathbf{x}) t^{n-\ell}
$$

and

$$
P_{B}(t)=\prod_{j=1}^{n}\left(t-y_{j}\right)=\sum_{j=0}^{m}(-1)^{j} \sigma_{j}(\mathbf{y}) t^{m-j} .
$$

Let

$V_{k, \ell}=\left[\begin{array}{c}1 \\ x_{k} \\ x_{k}^{2} \\ \vdots \\ x_{k}^{n-1} \\ y_{\ell} \\ x_{k} y_{\ell} \\ \vdots \\ x_{k}^{n-1} y_{\ell} \\ \vdots \\ y_{\ell}^{m-1} \\ x_{k} y_{\ell}^{n-1} \\ \vdots \\ x_{k}^{n-1} y_{\ell}^{n-1}\end{array}\right]$

and let $\bar{A}$ be the $n \times n$ matrix,

$$
\bar{A}=\left[\begin{array}{ccccccc}
0 & 1 & 0 & 0 & 0 & \cdots & 0 \\
0 & 0 & 1 & 0 & 0 & \cdots & 0 \\
0 & 0 & 0 & 1 & 0 & \cdots & 0 \\
\vdots & \vdots & \vdots & \vdots & \vdots & & \vdots \\
(-1)^{n+1} \sigma_{n}(\mathbf{x}) & (-1)^{n} \sigma_{n-1}(\mathbf{x}) & & & & \cdots & \sigma_{1}(\mathbf{x})
\end{array}\right]
$$

Now let $A$ be the following $n m \times n m$ matrix

$$
A=\left[\begin{array}{lllll}
\bar{A} & & & & \\
& \bar{A} & & & \\
& & \bar{A} & & \\
& & & \ddots & \\
& & & & \bar{A}
\end{array}\right] .
$$


By plugging $x_{k}$ into $P_{A}(t)$, we find that

$$
x_{k}^{n}=\sum_{\ell=1}^{n}(-1)^{\ell+1} \sigma_{\ell}(\mathbf{x}) x_{k}^{n-1} .
$$

This implies that

$$
A V_{k, \ell}=x_{k} \cdot V_{k, \ell}
$$

where $A$ is independent of $k$ and $\ell$. In a similar way we can construct a matrix, $B$, with entries given by the elementary symmetric functions such that

$$
B V_{k, \ell}=y_{\ell} V_{k, \ell} .
$$

Now

$$
\begin{aligned}
A B V_{k, \ell} & =A y_{\ell} V_{k, \ell} \\
& =y_{\ell} A V_{k, \ell} \\
& =x_{k} y_{\ell} V_{k, \ell} .
\end{aligned}
$$

Thus $\left\{x_{k} y_{\ell}\right\}$ are $n m$ distinct roots of $\operatorname{det}(t I-A B)$ which is a monic polynomial of degree $n m$. Therefore,

$$
\operatorname{det}(t I-A B)=\prod_{i=1}^{n} \prod_{j=1}^{m}\left(t-x_{i} y_{j}\right) .
$$

If the $x_{k}$ 's and $y_{\ell}$ 's were not independent variables, we would not be able to conclude that the elements in $\left\{x_{k} y_{\ell}\right\}$ are distinct.

With this lemma, we are ready to prove that the set of totally real numbers form a field under the operation $x \mapsto \sqrt{1+x^{2}}$.

Theorem. $\mathbb{F}_{\sqrt{1+x^{2}}} \subset \mathbb{F}_{T R}$.

Proof. If $\alpha, \beta \in \mathbb{F}_{T R}$, we must show that $-\alpha, \alpha^{-1}, \sqrt{1+\alpha^{2}}, \alpha+\beta, \alpha \cdot \beta \in \mathbb{F}_{T R}$. Let $\left\{\alpha_{i}\right\}_{i=1}^{n}$ be the conjugates of $\alpha$ and $\left\{\beta_{j}\right\}_{j=1}^{m}$ be the conjugates of $\beta$. We will prove the 
theorem by considering the following five polynomials.

$$
\begin{aligned}
q_{-\alpha}(t) & =\prod_{i=1}\left(t+\alpha_{i}\right), \\
q_{\alpha^{-1}}(t) & =\left(\prod_{i=1}^{n}\left(t-\alpha_{i}^{-1}\right)\right)\left(\prod_{i=1}^{n} \alpha_{i}\right), \\
q_{\sqrt{1+\alpha^{2}}}(t) & =\prod_{i=1}^{n}\left(t^{2}-1-\alpha_{i}^{2}\right), \\
q_{\alpha+\beta}(t) & =\prod_{i=1}^{n} \prod_{j=1}^{m}\left(t-\alpha_{i}-\beta_{j}\right), \\
q_{\alpha \beta}(t) & =\prod_{i=1}^{n} \prod_{j=1}^{m}\left(t-\alpha_{i} \beta_{j}\right) .
\end{aligned}
$$

The proofs of the first three cases are similar, and the proofs of the last two cases are similar, so we will only prove, in detail, the third case and the fifth case. If we expand $q_{\sqrt{1+\alpha^{2}}}(t)$, it is clear that the coefficients of $t^{k}$ will be symmetric polynomials in the $\alpha_{i}$. They may, therefore, be expressed as rational polynomials in the elementary symmetric polynomials of the $\alpha_{i}$. Since $(-1)^{\ell} \sigma_{\ell}(\boldsymbol{\alpha})$ are the coefficients of the minimal polynomial for $\alpha$ we may conclude that $q_{\sqrt{1+\alpha^{2}}}(t) \in \mathbb{Q}[t]$. It is clear that $\sqrt{1+\alpha^{2}}$ is a root of $q_{\sqrt{1+\alpha^{2}}}(t)$, thus the minimal polynomial of $\sqrt{1+\alpha^{2}}, p_{\sqrt{1+\alpha^{2}}}(t)$, divides $q_{\sqrt{1+\alpha^{2}}}(t)$. The fact that $\alpha$ is totally real implies that all of the conjugates, $\alpha_{i}$, are real. Thus, $1+\alpha_{i}^{2}$ are all real and positive, so $\pm \sqrt{1+\alpha_{i}^{2}}$ are all real. We now conclude that all of the roots of $q_{\sqrt{1+\alpha^{2}}}(t)$ are real, and therefore $\sqrt{1+\alpha^{2}}$ is totally real.

For the fifth case, we use the previous lemma to conclude that $q_{\alpha \beta}(t) \in \mathbb{Q}[t]$. Clearly, $\alpha \beta$ is a root of $q_{\alpha \beta}(t)$ and all of the roots of $q_{\alpha \beta}(t)$ are real because $\alpha$ and $\beta$ are totally real. In the other three cases, it is necessary to show that each of the $q$ 's are polynomials with rational coefficients and only real roots. The first two cases may be tackled with the fact that the elementary symmetric polynomials generate the algebra of all symmetric polynomials. The fourth case may be verified with a lemma analogous to the previous lemma stating that

$$
\prod_{i=1}^{n} \prod_{j=1}^{m}\left(t-x_{i}-y_{j}\right)=\operatorname{det}(t I-A-B) .
$$

This theorem gives us a practical way to decide that certain shapes may not be constructed using origami. For example, it is not possible using origami, to construct 
two cubes such that the volume of the second cube is twice that of the first cube. If this construction were possible, $\sqrt[3]{2}$ would be an origami number and would therefore be totally real. One, however, finds that the conjugates of $\sqrt[3]{2}$ are $\sqrt[3]{2}\left(-\frac{1}{2} \pm \frac{\sqrt{3}}{2} i\right)$ and $\sqrt[3]{2}$, but the first two are not real, so $\sqrt[3]{2}$ is not an origami number.

As we have seen before, $\sqrt{2}=\sqrt{1+1^{2}}$ and $\sqrt{4+2 \sqrt{2}}=\sqrt{1+(1+\sqrt{2})^{2}}$ are origami numbers, so $\sqrt{2+\sqrt{2}}=\sqrt{2}^{-1} \sqrt{4+2 \sqrt{2}}$ is an origami number. ¿From this we see the following corollary.

Corollary. It is not possible to construct a right triangle with arbitrarily given hypotenuse and leg using origami.

Proof. If this were possible, it would be possible to construct a right triangle with hypotenuse $\sqrt{2+\sqrt{2}}$ and leg 1 , since these are origami numbers. Any such triangle would have a leg of length $\sqrt{1+\sqrt{2}}=\sqrt{(\sqrt{2+\sqrt{2}})^{2}-1^{2}}$, but this is impossible because $\sqrt{1+\sqrt{2}}$ is not totally real.

The following corollary is a consequence of the standard algebraic description of compass and straight edge constructions and the two previous theorems $[\mathrm{AH}]$.

Corollary. Every thing which is constructible with origami is constructible with a compass and straight edge, but the converse is not true.

We want to expand on the relationship between compass and straight edge constructions and origami constructions. To review, compass and straight edge constructions, let $\mathbb{F}_{\sqrt{x}}$ be the smallest subfield of $\mathbb{C}$ closed under the operation $x \mapsto \sqrt{x}$, then $\mathbb{F}_{\sqrt{x}} \cap \mathbb{R}$ is the collection of numbers which are constructible with a compass and straight edge. ¿From our work thus far, it is evident that the origami numbers, $\mathbb{F}_{0}$, are contained in $\mathbb{F}_{\sqrt{x}} \cap \mathbb{F}_{T R}$. It is in fact the case that $\mathbb{F}_{0}=\mathbb{F}_{\sqrt{x}} \cap \mathbb{F}_{T R}$. This characterization of the origami numbers is related to David Hilbert's $17^{\text {th }}$ problem. At the International Congress of Mathematics at Paris in 1900, Hilbert gave a list of 23 problems [B]. His $17^{\text {th }}$ problem was to show that any rational function which is non-negative when evaluated at any rational number is a sum of squares of rational functions. In 1926, Artin solved Hilbert's $17^{\text {th }}$ problem [Ar]. The key idea which Artin used was the notion of totally positive. An element of a field is defined to be totally positive if it is positive in every order on the field. Artin proved that an element is totally positive if and only if it is a sum of squares. This is the idea which 
we use to prove the final characterization of the origami numbers.

Fact. [L, page 457]. If $K$ is a finite real algebraic extension of $\mathbb{Q}$, then an element of $K$ is a sum of squares in $K$ if and only if all of its real conjugates are positive.

Theorem. $\mathbb{F}_{0}=\mathbb{F}_{\sqrt{1+x^{2}}}=\mathbb{F}_{\sqrt{x}} \cap \mathbb{F}_{T R}$.

Proof. We have already shown that $\mathbb{F}_{0}=\mathbb{F}_{\sqrt{1+x^{2}}}$ and that $\mathbb{F}_{0} \subset \mathbb{F}_{\sqrt{x}} \cap \mathbb{F}_{T R}$, so we need to show that $\mathbb{F}_{\sqrt{x}} \cap \mathbb{F}_{T R} \subset \mathbb{F}_{\sqrt{1+x^{2}}}$. If $\alpha \in \mathbb{F}_{\sqrt{x}} \cap \mathbb{F}_{T R}$, then there exists a sequence of totally real numbers, $\left\{\beta_{i}\right\}_{i=1}^{n}$ and a sequence of totally real fields $\left\{K_{j}\right\}_{j=0}^{n-1}$ such that $K_{0}=\mathbb{Q}$, $K_{i}=K_{i-1}\left(\beta_{k}\right), \alpha=\beta_{n}$, and each $\beta_{i}$ has degree 2 over $K_{i-1}$. Since $\beta_{i}$ has degree 2 over $K_{i-1}, \beta_{i}$ is a root of a polynomial of the form

$$
x^{2}+c_{i} x+d_{i}
$$

where $c_{i}, d_{i} \in K_{i-1}$. Therefore, $\left(\beta_{i}+c_{i} / 2\right)^{2}=c_{i}^{2} / 4-d_{i}$. By the proof of the previous theorem, we know that every conjugate of $\left(\beta_{i}+c_{i} / 2\right)^{2}$ is the square of some conjugate of $\beta_{i}+c_{i} / 2$. Hence, each of the conjugates of $\left(\beta_{i}+c_{i} / 2\right)^{2}$ are positive and $\left(\beta_{i}+c_{i} / 2\right)^{2}$ is a sum of squares of elements in $K_{i-1}$. Say that

$$
\left(\beta_{i}+c_{i} / 2\right)^{2}=r_{i, 1}^{2}+r_{i, 2}^{2}+\cdots+r_{i, m}^{2}
$$

then,

$$
\beta_{i}=r_{i, 1} \sqrt{1+\left[\frac{r_{i, 2}}{r_{i, 1}} \sqrt{1+\left[\frac{r_{i, 3}}{r_{i, 2}} \sqrt{\cdots}\right]^{2}}\right]^{2}}-\frac{c_{i}}{2}
$$

and we are done. This shows that any totally real number in $\mathbb{F}_{\sqrt{x}}$ is an origami number.

Legend has it that the ancient Athenians were faced with a plague. In order to remedy the situation, they sent a delegation to the oracle of Apollo at Delos. This delegation was told to double the volume of the cubical altar to Apollo. However, the Athenians doubled the length of each side of the altar, thereby creating an altar with eight times the volume rather than twice the volume of the original altar. Needless to say, the plague only got worse. For years, people have tried to double the size of a cube with compass and straight edge, and the gods have not smiled upon them. We now can see that the gods will not be satisfied with our elementary origami either. 


\section{References}

[AH] G. Alexanderson, A. Hilman, "A First Undergraduate Course in Abstract Algebra," 3rd edition, Wadsworth Publishing Company, 1983.

[Ar] E. Artin, Über die zerlegung definiter funktionen in quadrate, Abh. Math. Sem. Hausischen Univ. 5 (1927), 100-115.

[B] F. Browder, Mathematical developments arising from Hilbert problems, in "Proceedings of Symposia in Pure Mathematics," Volume 28, American Mathematical Society, 1976.

[K] F. Klein, Vortrage über ausgewahlte Fragen der Elementargeometrie, Teubner, 1895.

[L] S. Lang, "Algebra," 3rd edition, Addison-Wesley, 1993.

[M] J. Montroll, "Origami for the Enthusiast," Dover, 1979.

[R] T. Sundara Row, Geometric Exercises in Paper Folding, Dover, 1966. 